\title{
Aplicaciones en energía solar
}

\section{8}

DOI: $10.31978 / 014-18-009-X .38$

DANIEL MASA Bote

AKKA Technologies, Madrid, España

ANA CAROLINA Do AMARAL BURGHi y TOBIAS HiRSCH

German Aerospace Center (DLR), Institute of Solar Research, Stuttgart, Alemania

ROBERT PITZ-PAAL

German Aerospace Center (DLR), Institute of Solar Research, Colonia, Alemania

El uso de energía solar no ha sido desarrollado porque la industria del petróleo no posee el sol.

RALPH NADER

Las actividades relacionadas con la obtención de energía en las que las condiciones meteorológicas tengan un impacto crítico pueden beneficiarse de importantes mejoras en su rendimiento si las previsiones meteorológicas son acertadas. Con el surgimiento de la predicción probabilista este beneficio puede ser aún mayor pues, como se describe en otras partes de este libro y más adelante en este mismo capítulo, pueden tomarse mejores decisiones si se conocen las probabilidades asociadas a la superación de distintos umbrales de ocurrencia de eventos meteorológicos. Utilizando el lenguaje económico, usar predicciones probabilistas tiene un valor relativo mayor, si se saben utilizar y serán, por tanto, más rentables. Nuestro futuro sólo puede encontrarse en la energía sostenible, como es el caso de la solar o la eólica.

Palabras clave: sistemas de predicción por conjuntos aplicados a la energía solar, predicción probabilista para la previsión de energía solar fotovoltaica, efecto cartera, almacenamiento eléctrico, gestión de la demanda, inclusión de incertidumbres de predicciones meteorológicas en la optimización del despacho de energía por plantas solares de concentración.

Imagen parte superior: la torre solar Jülich es un modelo de investigación para actuales y futuras centrales térmicas solares comerciales. Los experimentos se llevan a cabo con radiación solar altamente concentrada. Fotografía: DLR. 


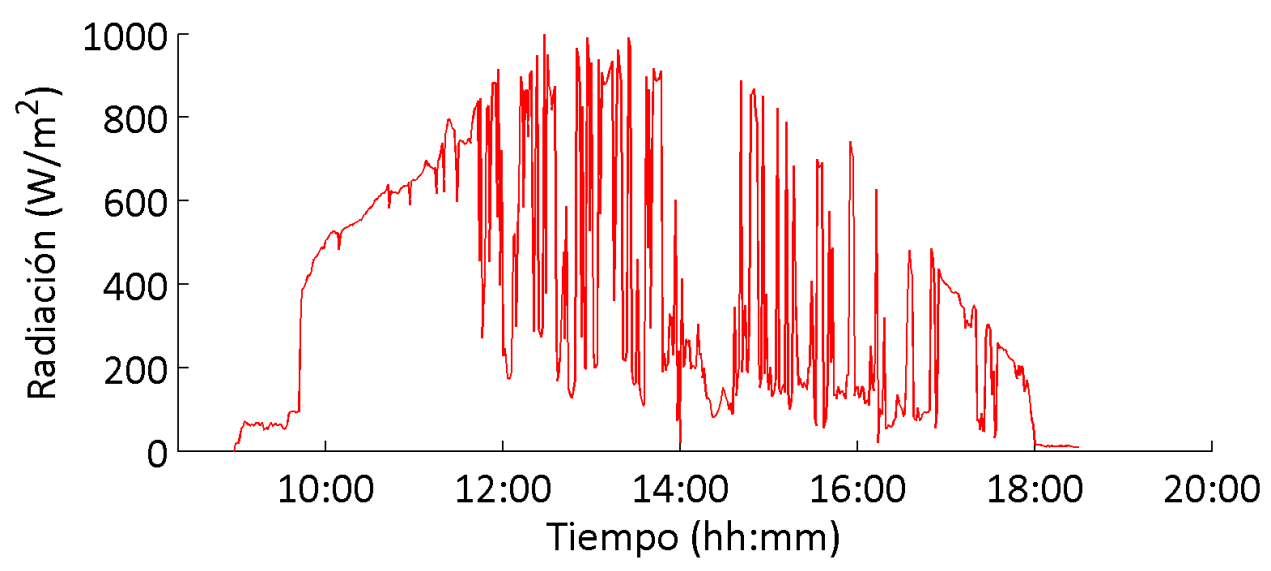

Figura 38.1: Variación de la radiación incidente en un día de nubosidad variable.

\subsection{Previsiones meteorológicas en la energía solar fotovoltaica}

DANIEL MASA Bote

AKKA Technologies, Madrid, España

La afirmación con la que termina el encabezado del capítulo es, si cabe, más relevante en la situación actual de creciente penetración de las energías renovables en las redes eléctricas. De acuerdo con la Agencia Internacional de la Energía, la energía solar fotovoltaica contribuyó con $1 \%$ al total de la energía eléctrica producida en todo el mundo. Esta penetración, pese a parecer baja a primera vista, está desigualmente distribuida: alcanzando un $8.1 \%$ en Italia y un $6.0 \%$ en Alemania, por ejemplo. En España la contribución de la energía solar fotovoltaica en ese año fue del $3.1 \%$. Además de la creciente preocupación por los efectos ambientales provocados por fuentes tradicionales de energía, basadas en combustibles fósiles, y por su inevitable agotamiento, este crecimiento ha estado provocado, además, por el abaratamiento de los costes de producción de las energías renovables, siendo competitivas en coste con las fuentes convencionales de generación en múltiples países. Es esperable que en el futuro esta tendencia no solo se mantenga sino que se acreciente.

La energía solar fotovoltaica, al igual que otras energías renovables como la eólica, ha sido considerada tradicionalmente una fuente de energía no fiable debido a su dependencia para producir energía de las condiciones meteorológicas, principalmente la radiación en el caso de los sistemas fotovoltaicos al estar íntimamente ligada su generación a la radiación incidente. La integración de la energía solar fotovoltaica en las redes eléctricas parte por lo tanto de la capacidad de conocer de antemano la capacidad de producción de los sistemas fotovoltaicos.

Las predicciones de radiación incidente se pueden agrupar en dos grupos bien diferenciados en función del horizonte de predicción o anticipación con la que se desea conocer la generación futura: predicciones a corto plazo con un horizonte de predicción de 24 a 48 horas y predicciones a muy corto plazo o predicciones inmediatas con las que se desea conocer las fluctuaciones de la generación de los sistemas fotovoltaicos con una antelación que va desde las 3 horas hasta periodos de unos pocos minutos.

Estos dos horizontes de predicción responden a dos necesidades concretas de los sistemas eléctricos. En el caso de predicciones a $24-48$ horas, es la necesidad por parte de los operadores de los sistemas eléctricos de conocer con suficiente anticipación la capacidad de todos los generadores del sistema de tal manera que el operador pueda despachar la producción individual de cada generador teniendo en cuenta la demanda. Las predicciones inmediatas o a muy corto plazo están orientadas a contribuir a la estabilidad de la red eléctrica limitando fluctuaciones muy rápidas en la energía generada por un sistema fotovoltaico. A modo de ejemplo, en la Figura 38.1 se muestra la variación en la radiación durante un día de nubosidad variable.

Las predicciones a muy corto plazo quedan fuera del ámbito del presente estudio. No obstante, cabe indicar que estas predicciones no suelen efectuarse a partir 
de modelos numéricos de predicción sino a partir de modelos estadísticos o análisis y extrapolación de imágenes obtenidas por satélite o radar.

\subsubsection{Beneficios de las predicciones pro- babilistas}

Como ya se ha comentado, el objetivo de las predicciones a corto plazo es conocer de antemano y con la mayor exactitud posible cuánta energía va a generar un sistema fotovoltaico para que estos puedan participar en los despachos de energía realizados por los operadores de los sistemas eléctricos. En los despachos, el operador del sistema eléctrico asigna a cada generador cuánta energía debe producir en función de su capacidad declarada de generación, que en el caso de los sistemas fotovoltaicos serán las predicciones a corto plazo, y la demanda prevista. Este criterio de asignación se basa tanto en criterios técnicos que buscan asegurar la fiabilidad y seguridad del suministro eléctrico como en criterios económicos que persiguen reducir los costes de generación de energía eléctrica. Los despachos de electricidad se realizan a escala horaria, para un día completo y con un mínimo de 12 horas de adelanto.

En la actualidad, el mecanismo más extendido para realizar el despacho de la generación eléctrica suele ser mediante mercados mayoristas diarios, también llamados pool, en los cuales se casan las ofertas de venta y compra de electricidad presentadas por productores y consumidores de tal manera que la demanda se cubra preferentemente con las fuentes de generación más económicas. En el caso de que un generador eléctrico se desvíe de la cantidad que le ha sido asignada, puede incurrir en penalizaciones económicas. Lo más habitual es que solamente se penalice a los productores si las desviaciones incurridas son contrarias al sistema, es decir, si se ha producido menos energía de la declarada en un momento en que la red era deficitaria (mayor consumo que generación) o viceversa.

En el caso de predicciones deterministas, la energía a declarar por los gestores de una huerta solar será directamente el resultado de la predicción y, por tanto, la probabilidad de incurrir en desvíos respecto a la capacidad declarada dependerá exclusivamente de la bondad del modelo de predicción que suele situarse en torno al $40 \%$ para valores horarios de radiación con horizonte de 24 horas. Por supuesto, siempre se puede declarar una energía menor que la predicción para tener un cierto margen de maniobra en caso de que la predicción falle por exceso. El caso de que la predicción falle por exceso se considera trivial pues basta con limitar la generación. No obstante, aunque este proceder pueda minimizar la probabilidad de incurrir en desvíos, no supone una gestión óptima del recurso solar pues de manera sistemática se está declarando una capacidad de producción menor a la real.

En el caso de disponer de predicciones de producción probabilistas, un ejemplo de las cuales se muestra en la Figura 38.2, acompañadas por tanto de sus correspondientes intervalos de confianza, es posible saber de antemano no solo la energía generada por el sistema sino tener una idea de la distribución de probabilidad del error asociado a dicha predicción.

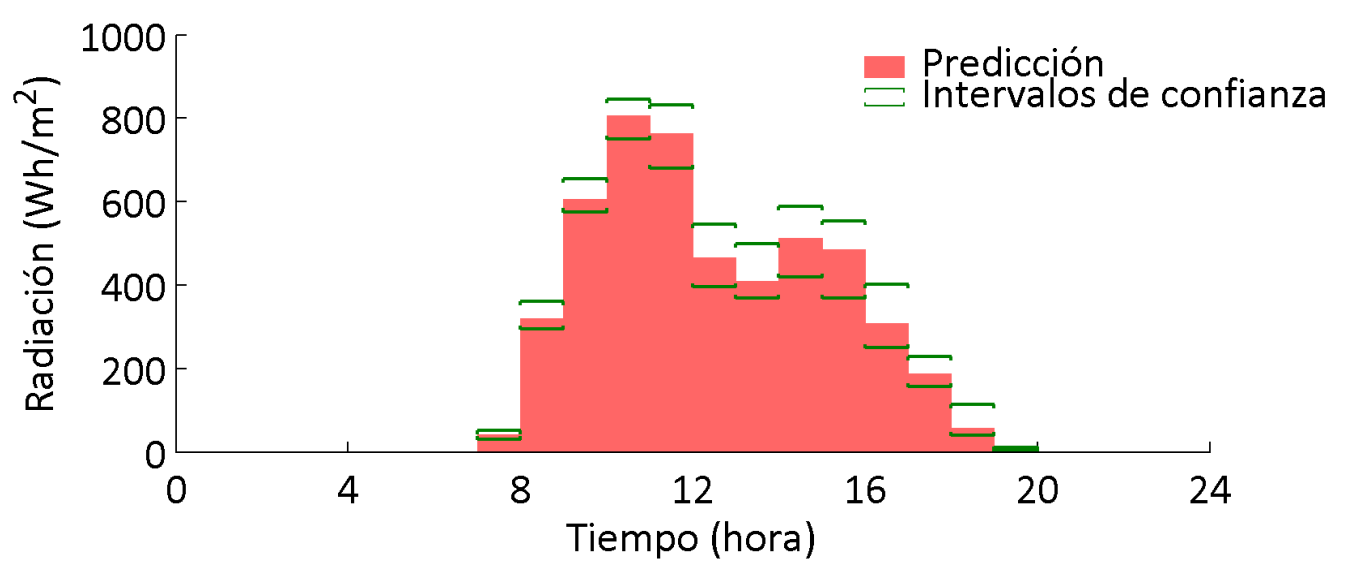

Figura 38.2: Predicción de valores horarios de radiación junto con intervalos de confianza. 
Con esta información es posible hacer una estimación más razonable y ajustada de la energía que se debe declarar de cara al sistema de tal manera que, a largo plazo, se consigan minimizar tanto las penalizaciones debidas a desvíos como la energía desaprovechada. La estimación de la energía a declarar puede afinarse si se dispone de información sobre errores de predicción cometidos en el pasado y es posible realizar una corrección estadística, empleando redes neuronales, por ejemplo, de la salida del modelo de predicción empleado.

\subsubsection{Efecto cartera, almacenamiento eléctrico y gestión de la demanda}

Como ya se ha comentado anteriormente, la integración efectiva de los sistemas fotovoltaicos en las redes eléctricas implica necesariamente, conocer con la mayor exactitud posible la energía que generarán estos sistemas a corto plazo. En el caso que nos ocupa de la generación fotovoltaica, las predicciones se caracterizan por su elevada incertidumbre (hasta un $40 \%$ ) y, a la espera de que se produzcan mejoras en los procedimientos predictivos que permitan reducir este error, en la industria se han propuesto soluciones alternativas que, en vez de reducir la incertidumbre asociada a los modelos de predicción, permiten paliar sus efectos.

La primera de estas soluciones es el denominado efecto cartera, consistente en agrupar la generación de varios sistemas fotovoltaicos como si constituyesen un único sistema, de tal manera que la energía generada por el conjunto sea más previsible que la de cada sistema individual. Para que la reducción de la incertidumbre sea efectiva es necesario que los sistemas se encuentren lo suficientemente distantes entre sí como para que la irradiación incidente en cada uno pueda considerarse independiente de las demás. A fin de ilustrar este efecto, se muestran en la Tabla 38.1 los errores cuadráticos medios cometidos al predecir valores horarios de radiación con un horizonte de predicción de 24 horas durante un año completo en cinco localidades de la península: Santander, Logroño, Soria, Madrid y Cáceres así como el resultado de la predicción conjunta, efecto cartera, para las cinco localidades. Puede observarse que el error cuadrático medio conjunto es inferior a todos los demás, consistentemente con la teoría expuesta en las secciones 13.6 .3 en la página 178 y 15.7 en la página 221 .

La siguiente solución alternativa propuesta consiste en combinar los sistemas fotovoltaicos con sistemas de almacenamiento, típicamente baterías eléctricas, siendo las más populares las de ion-litio. La idea detrás de combinar generación renovable y almacenamiento eléctrico es bastante sencilla: se usará el sistema de almacenamiento como un búfer en el que se inyectará energía cuando esta sea excedentaria y del cual se extraerá energía cuando esta sea deficitaria. El resultado es que el conjunto generador más batería se muestra de cara a la red como un generador cuya diferencia entre energía declarada y energía finalmente entregada a la red se ha desacoplado, gracias a la batería, del error de predicción. El principal inconveniente de esta solución es el elevado coste de los sistemas de almacenamiento.

A modo de ilustración se muestra en la Figura 38.3 en la página siguiente el efecto que tiene la inclusión de almacenamiento en un sistema fotovoltaico real de $7 \mathrm{~kW}$. Se han considerado distintos tamaños de batería en función de la capacidad de la misma o máxima energía que es capaz de almacenar, $C_{b a t, \max }$, y la potencia máxima de carga/descarga que es la máxima potencia que puede entregar de manera instantánea el sistema, $P_{b a t, \text { max }}$. Como es de esperar, a medida que aumenta el tamaño de la batería, tanto en capacidad como potencia máxima, se reduce la diferencia entre energía declarada y producida. En el mejor de los casos, esta diferencia tiene un error cuadrático medio, RMSE, de $397 \mathrm{Wh}$ mientras que el error cometido en la predicción, sin contar con el almacenamiento, es de $1087 \mathrm{Wh}$.

\begin{tabular}{|c|c|}
\hline Localidad & RMSE $\left[\mathrm{Wh} / \mathrm{m}^{2}\right]$ \\
\hline \hline Santander (I) & 186,3 \\
\hline Logroño (II) & 165,2 \\
\hline Soria (III) & 159,7 \\
\hline Madrid (IV) & 150,6 \\
\hline Cáceres (V) & 138,8 \\
\hline Conjunta & 106,6 \\
\hline
\end{tabular}

Tabla 38.1: Resultados de la predicción de la irradiación para cada localidad y para la combinación de las cinco localidades. 


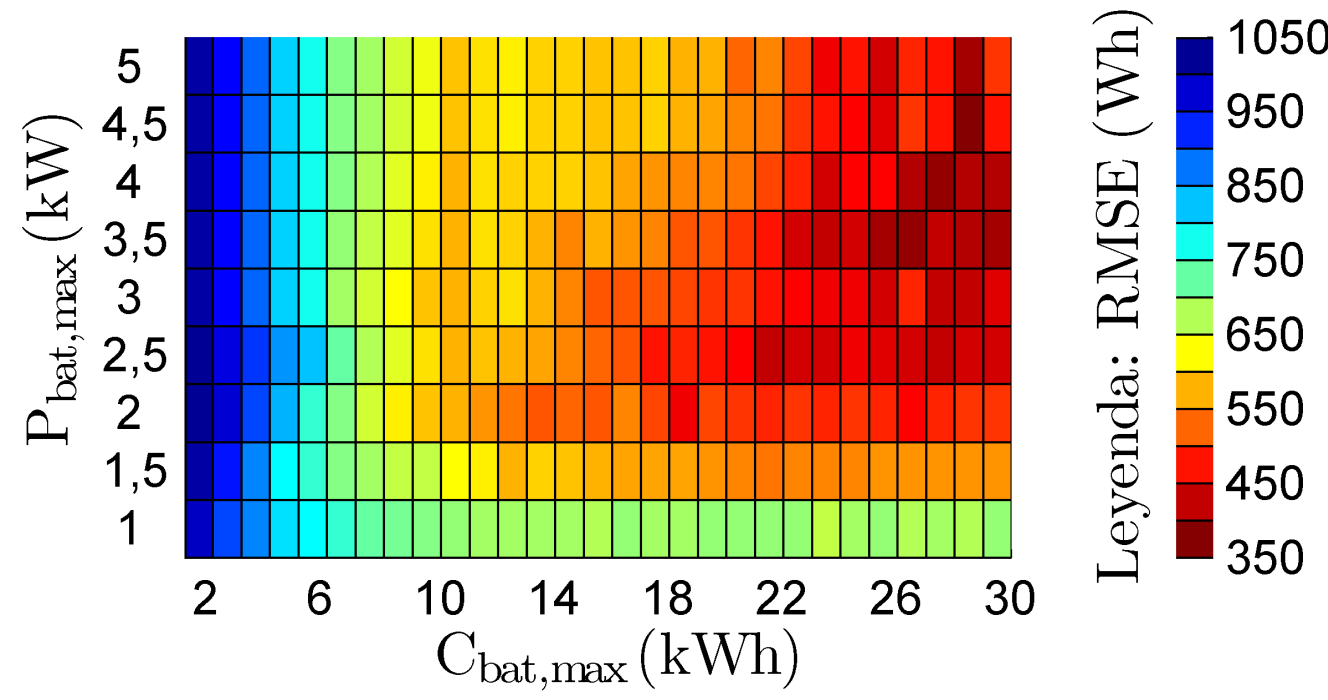

Figura 38.3: Diferencia entre energía declarada y producida en función del dimensionamiento del sistema de almacenamiento eléctrico.

El uso de almacenamiento eléctrico no solo permite mitigar los efectos de la incertidumbre en la predicción sino que también permite proporcionar servicios auxiliares a la red eléctrica que un sistema fotovoltaico, por sí solo, no es capaz. Por ejemplo, el perfil de generación de la Figura 38.1 en la página 600, aunque pudiese predecirse con total exactitud no es deseable para la red eléctrica debido al efecto negativo que tiene sobre el sistema su elevada variabilidad. La combinación de un sistema fotovoltaico con dicho perfil de generación y una batería con una estrategia de gestión adecuada permitirían aplanar dicho perfil de tal manera que, para la red eléctrica, el conjunto de generación fotovoltaica más almacenamiento sería el de un único generado estable en el tiempo.

La última solución posible para mitigar el efecto de la incertidumbre en la predicción es la combinación de generación fotovoltaica con sistemas de gestión de la demanda eléctrica. La gestión de la demanda eléctrica consiste en la capacidad por parte de los consumidores de modificar su consumo de energía eléctrica reduciendo el mismo o desplazándolo temporalmente. Esta solución implica que los sistemas fotovoltaicos se ubiquen en la proximidad de puntos de consumo como puede ser sector residencia, industria o comercio En este sentido, la gestión de la demanda se comportaría como la batería en el caso anterior: se desplazaría el consumo de electricidad de aquellos periodos en que la generación fotovoltaica es deficitaria a aquellos en que es excedentaria. Por supuesto, tanto almacenamiento eléctrico como gestión de la demanda no son mutuamente excluyentes y ambas estrategias pueden llevarse a cabo de manera conjunta. 


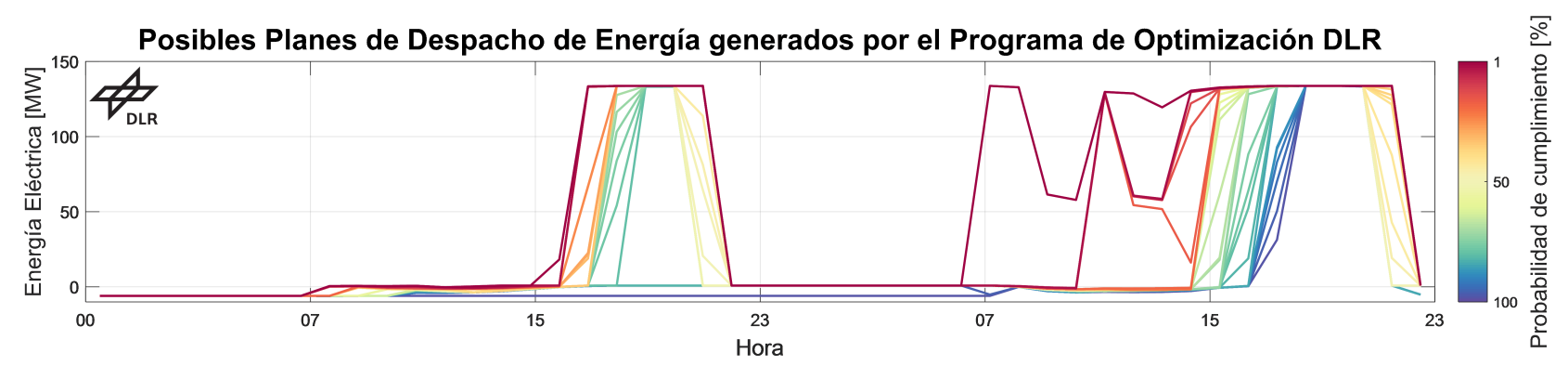

Figura 38.4: Rango de posibles planes de entrega de electricidad para dos días específicos, de acuerdo con la probabilidad de cumplir con la energía comprometida para ese periodo.

\title{
38.2 Inclusión de incertidumbres de predicciones meteorológicas en la op- timización del despacho de energía por plantas solares de concentra- ción
}

\author{
Ana Carolina do Amaral Burghi y Tobias Hirsch \\ German Aerospace Center (DLR), Institute of Solar Research, Wankelstrasse 5, 70563 Stuttgart, Alemania \\ ROBERT PITZ-PAAL \\ German Aerospace Center (DLR), Institute of Solar Research, Linder Höhe, 51147 Colonia, Alemania
}

La alta penetración de energías renovables en redes eléctricas ofrece la oportunidad de equilibrar la producción y la demanda de electricidad mediante plantas de Energía Solar de Concentración (CSP, en inglés Concentrated Solar Power [1]). Gracias a su capacidad de almacenamiento térmico, las plantas CSP proporcionan una cierta flexibilidad, y permiten desacoplar la recolección de energía térmica del sol y su transformación en energía eléctrica mediante un ciclo de potencia, por lo que hacen posible generar energía en el momento que más se necesita.

Para programar con precisión la producción de electricidad en los próximos días, el despacho de energía de estas plantas debe basarse en la demanda de electricidad y las predicciones meteorológicas [2]. Esta programación permite la participación activa de las plantas de CSP en el mercado de energía, reforzando su importancia en un futuro donde se vislumbra una alta integración y combinación de distintas fuentes de energía renovable.
El término despacho es el utilizado en el mercado eléctrico, refiriéndose al envío de energía de la planta eléctrica a la red, mientras que suministro está relacionado con la energía que va de la red a los consumidores. Son concepto muy similares, pero acercándose al detalle técnico se trata de distintas partes de un mismo proceso.

La atmósfera es un sistema caótico, y por ello es imposible predecir su estado futuro de forma exacta. Así es recomendable considerar la incertidumbre de estas en la planificación del despacho de energía por centrales CSP.

La modificación de entrega de energía ya programada es limitada y generalmente conlleva penalizaciones o precios reducidos. En consecuencia, para garantizar la optimización del despacho, es esencial considerar estas incertidumbres en el cronograma de la entrega de energía, considerando su dependencia con el método y la precisión de los pronósticos meteorológicos. 
La precisión de las predicciones meteorológicas, realizadas por diferentes métodos de pronóstico, es un tema relevante que ayuda a evaluar su impacto en la programación del despacho de energía, así como en el ingreso económico de las plantas CSP.

La Agencia Aeroespacial Alemana (Deutsches Zentrum für Luft- und Raumfahrt e.V., DLR) desarrolló un programa de optimización de despacho de energía eléctrica, utilizado para planear los cronogramas de operación de una planta CSP en el mercado diario de energía. Este programa considera los pronósticos meteorológicos y los precios de la electricidad, con un enfoque especial en la incorporación de incertidumbres.

La estrategia del programa de DLR se basa en un cálculo que divide el algoritmo de optimización del procesamiento de la incertidumbre, posibilitando así la utilización de predicciones meteorológicas probabilistas.

El algoritmo de optimización es ejecutado directamente con todos los escenarios meteorológicos posibles, en el caso de que la predicción sea probabilista, o con la predicción determinista y sus niveles de probabilidad, mientras que la incertidumbre se trata como un pos proceso.

Este posproceso consiste en la categorización de los escenarios optimizados según un análisis de riesgos del cumplimiento de la entrega de energía. Por lo tanto, el resultado de la optimización es un rango de posibles planes de despacho de energía (Figura 38.4 en la página anterior), y el procesamiento posterior de la incertidumbre puede categorizar esos posibles planes, de acuerdo con el riesgo de cumplir con la entrega de energía comprometida.

El enfoque del programa desarrollado por DLR brinda la posibilidad de incluir la incertidumbre representada por predicciones meteorológicas probabilistas en combinación con otros parámetros del mercado eléctrico. Por lo tanto, pueden elaborarse estrategias exclusivas de entrega de energía, de acuerdo con la calidad de las predicciones meteorológicas, las características del mercado y el enfoque de operación de la planta CSP.

Estas estrategias permiten aprovechar de forma óptima toda la información contenida en las predicciones probabilistas específicamente para plantas CSP. El valor económico relativo se presenta con detalle en la sec. 15.10 en la página 234 y es en estas aplicaciones donde puede calcularse para mostrar que el uso de SPC puede ser rentable con respecto a usar modelos deterministas.

Esta herramienta tiene como ventaja la utilización de pronósticos meteorológicos tanto deterministas como probabilistas, así como la evaluación de la calidad y eficiencia de éstos para optimizar el despacho de energía por plantas CSP.

La utilización de predicciones meteorológicas como entrada, especialmente la de tipo probabilista, puede permitir un aumento en los beneficios económicos y en la rentabilidad de estas plantas. Además, se prevé que los resultados relacionados con la exactitud en la predicción del tiempo, así como los posibles incrementos de ingresos financieros por operación de plantas CSP puedan ser de gran relevancia para una mejor comprensión sobre cómo emplear las incertidumbres para una óptima generación de energía. 


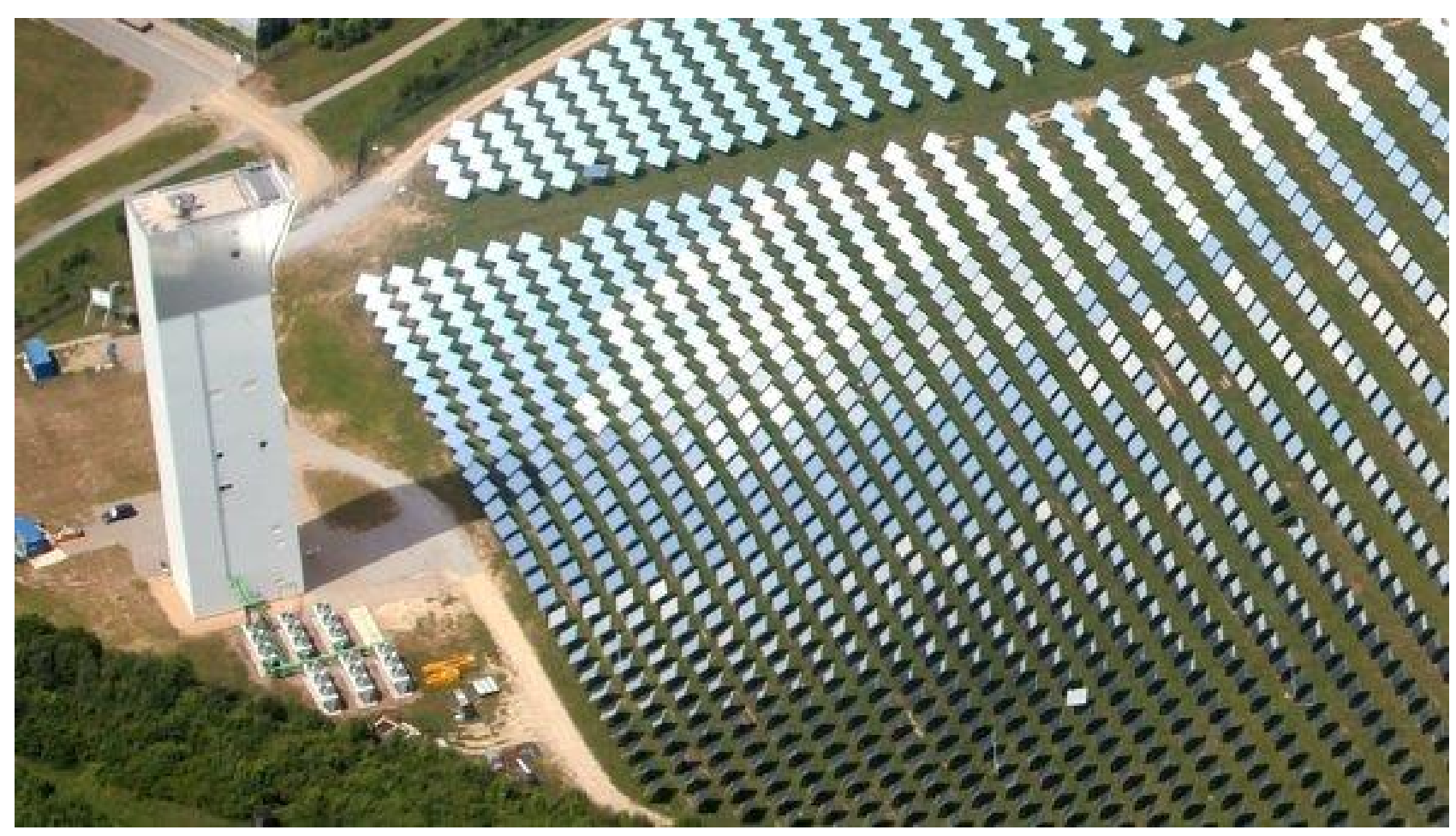

Figura 38.5: La torre solar JÜLICH es un modelo de investigación para actuales y futuras centrales térmicas solares comerciales. Los experimentos se llevan a cabo con radiación solar altamente concentrada. Fotografía: vista aérea de la torre solar, DLR. 


\subsection{Referencias}

[1] Burghi, Ana Carolina do amaral, Hirsch, Tobias y PITZ-PAAL, Robert. "CSP Dispatch Optimization considering Forecasts Uncertainties". En: SolarPACES. Chile, sep. de
2017. URL: http : / / elib . dlr . de / 114738/ (citado en página 604).

[2] VV. AA. IRENA - Adapting Market Design to High Shares of Variable Renewable Energy. Informe técnico. Abu Dhabi: International Renewable Energy Agency (IRENA), 2017 (citado en página 604). 